\title{
Deformed quasiparticle random phase approximation formalism for single- and two-neutrino double $\beta$ decay
}

\author{
R. Álvarez-Rodríguez, P. Sarriguren, and E. Moya de Guerra \\ Instituto de Estructura de la Materia, Consejo Superior de Investigaciones Científicas, Serrano 123, E-28006 Madrid, Spain \\ L. Pacearescu and Amand Faessler \\ Institut für Theoretische Physik, Universität Tübingen, D-72076 Tübingen, Germany \\ F. Šimkovic \\ Department of Nuclear Physics, Comenius University, SK-842 15 Bratislava, Slovakia \\ (Received 12 February 2004; revised manuscript received 23 September 2004; published 13 December 2004)
}

\begin{abstract}
We use a deformed quasiparticle random phase approximation formalism to describe simultaneously the energy distributions of the single $\beta$ Gamow-Teller strength and the two-neutrino double $\beta$ decay matrix elements. Calculations are performed in a series of double $\beta$ decay partners with $A=48,76,82,96,100,116$, $128,130,136$, and 150, using deformed Woods-Saxon potentials and deformed Skyrme Hartree-Fock mean fields. The formalism includes a quasiparticle deformed basis and residual spin-isospin forces in the particlehole and particle-particle channels. We discuss the sensitivity of the parent and daughter Gamow-Teller strength distributions in single $\beta$ decay, as well as the sensitivity of the double $\beta$ decay matrix elements to the deformed mean field and to the residual interactions. Nuclear deformation is found to be a mechanism of suppression of the two-neutrino double $\beta$ decay. The double $\beta$ decay matrix elements are found to have maximum values for about equal deformations of parent and daughter nuclei. They decrease rapidly when differences in deformations increase. We remark on the importance of a proper simultaneous description of both double $\beta$ decay and single Gamow-Teller strength distributions. Finally, we conclude that for further progress in the field, it would be useful to improve and complete the experimental information on the studied Gamow-Teller strengths and nuclear deformations.
\end{abstract}

DOI: 10.1103/PhysRevC.70.064309

PACS number(s): 21.60.Jz, 23.40.Hc, 23.40.Bw

\section{INTRODUCTION}

The recent experimental confirmation of neutrino oscillations has reinforced the interest in nuclear processes involving neutrinos; see [1] and references therein. Nuclear double $\beta$ decay is a rare second-order weak interaction process that takes place when the transition to the intermediate nucleus is energetically forbidden or highly retarded. Two main decay modes are expected in this process: The two-neutrino mode, involving the emission of two electrons and two neutrinos, and the neutrinoless mode with no neutrino leaving the nucleus. While the first type of process is perfectly compatible with the Standard Model, the second one violates lepton number conservation and its observation is linked to the existence of a massive Majorana neutrino. For this reason, considerable experimental and theoretical effort is being devoted to the study of this process [2].

From the theoretical point of view, one particular source of uncertainty is the evaluation of the nuclear matrix elements involved in the process. They have to be calculated as accurately as possible to obtain reliable estimates for the limits of the double $\beta$ decay half-lives. Since the nuclear wave functions and the underlying theory for treating the neutrinoless and the two-neutrino modes are similar, the usual procedure is to test first the nuclear structure component of the two-neutrino mode against the available experimental information on half-lives.

The proton-neutron quasiparticle random phase approximation (pnQRPA or QRPA in short) is one of the most reli- able and widely used microscopic approximations for calculating the correlated wave functions involved in $\beta$ and double $\beta$ decay processes. The method was first studied in Ref. [3] to describe the $\beta$ strength and was also successfully applied to the description of double $\beta$ decay [4] after the inclusion of a particle-particle $(p p)$ residual interaction, in addition to the particle-hole $(p h)$ usual channel. Many more extensions of the QRPA method have been proposed in the literature; see Ref. [5] and references therein.

An extension of the pnQRPA method to deal with deformed nuclei was done in Ref. [6], where a Nilsson potential was used to generate single-particle orbitals. Subsequent extensions including Woods-Saxon type potentials [7], residual interactions in the particle-particle channel [8], selfconsistent deformed Hartree-Fock (HF) mean fields with consistent residual interactions [9], and self-consistent approaches in spherical neutron-rich nuclei [10], can also be found in the literature. In Refs. $[9,11], \beta$-decay properties were studied on the basis of a deformed self-consistent HF + BCS + QRPA calculation with density-dependent effective interactions of Skyrme type. A deformed QRPA approach based on a phenomenological deformed Woods-Saxon potential was used to calculate the Gamow-Teller strength distributions for the two decay branches in double $\beta$ decay of ${ }^{76} \mathrm{Ge}[11,12]$.

Recently, the issue of nuclear deformation, which has usually been ignored in the QRPA-like treatments of nearly spherical nuclei, was raised in Refs. [12,13]. In Ref. [12], it 
was found that differences in deformation between the initial and final nuclei can have large effects on the double $\beta$ decay half-lives. Within the deformed QRPA using an axially symmetric Woods-Saxon single-particle basis, the particular case of the two-neutrino double $\beta$ decay ( $2 \nu \beta \beta$-decay) of ${ }^{76} \mathrm{Ge}$ was analyzed [12]. The effect of deformation on the other double $\beta$ decay processes of experimental interest has not been sufficiently studied yet, see $[12,14,15]$ and references therein.

In this work, we extend these deformed calculations $[9,12]$ by studying first the single $\beta$ branches that build up the double $\beta$ process and then the $2 \nu \beta \beta$-decay process itself. We focus on the $\beta^{-}$Gamow-Teller (GT) transitions of the double $\beta$ emitters as well as on the $\beta^{+}$Gamow-Teller transitions of the daughter nuclei ending up at the same intermediate virtual nucleus. The cases considered are those where the two-neutrino double $\beta$ decay half-lives have been measured, namely

$$
\begin{gathered}
{ }^{48} \mathrm{Ca} \rightarrow{ }^{48} \mathrm{Ti} ; \quad{ }^{76} \mathrm{Ge} \rightarrow{ }^{76} \mathrm{Se} ; \\
{ }^{82} \mathrm{Se} \rightarrow{ }^{82} \mathrm{Kr} ; \quad{ }^{96} \mathrm{Zr} \rightarrow{ }^{96} \mathrm{Mo} ; \\
{ }^{100} \mathrm{Mo} \rightarrow{ }^{100} \mathrm{Ru} ; \quad{ }^{116} \mathrm{Cd} \rightarrow{ }^{116} \mathrm{Sn} ; \\
{ }^{128} \mathrm{Te} \rightarrow{ }^{128} \mathrm{Xe} ; \quad{ }^{130} \mathrm{Te} \rightarrow{ }^{130} \mathrm{Xe} ; \\
{ }^{136} \mathrm{Xe} \rightarrow{ }^{136} \mathrm{Ba} ; \quad{ }^{150} \mathrm{Nd} \rightarrow{ }^{150} \mathrm{Sm} .
\end{gathered}
$$

In Sec. II, we present a brief summary containing the basic points in our theoretical description. Section III contains the results obtained for the ground-state properties of the nuclei mentioned above. In Sec. IV, we present our results for the GT strength distributions and discuss their dependence on the deformed mean field and residual interactions. Section V contains the results for the two-neutrino double $\beta$ decay calculations. The conclusions are given in Sec. VI.

\section{BRIEF DESCRIPTION OF THE THEORY}

In this section, we summarize briefly the theoretical formalism used to describe the Gamow-Teller transitions. More details can be found in Refs. [9,12,11].

The single-particle energies and wave functions are generated from two different methods to construct the deformed mean field, which is assumed to be axially symmetric. In one case, we start from a deformed Woods-Saxon (WS) potential. The parameters of this potential are taken from Ref. [16]. The isospin dependence of this parametrization allows one to extend it to any mass region. Previous QRPA calculations have shown that it provides a realistic description of the ground-state properties of deformed nuclei as well as good results on $M 1$ excitations [17] for nuclei in various mass regions. The quadrupole deformation of the WS potential is determined by fitting the microscopically calculated quadrupole moment to the corresponding experimental value. The hexadecapole deformation is expected to be small for these nuclei and we assume it is equal to zero.
In the other method, we follow a self-consistent HartreeFock procedure to generate microscopically the deformed mean field. This is done with density-dependent effective interactions of Skyrme type. Contrary to the previous case, the equilibrium deformation of the nucleus is obtained now self-consistently as the shape that minimizes the energy of the nucleus. In this work, we present the results obtained with the most common of the Skyrme forces, Sk3 [18], although sometimes we also show for comparison results obtained with the force SG2 [19].

In both schemes, WS and HF, the single-particle wave functions are expanded in terms of the eigenstates of an axially symmetric harmonic oscillator in cylindrical coordinates, using 11 major shells in the expansion. Pairing correlations between like nucleons are included similarly in both cases in the BCS approximation with fixed gap parameters for protons and neutrons. The gap parameters are determined phenomenologically from the odd-even mass differences through a symmetric five-term formula involving the experimental binding energies. The values obtained from this procedure for the nuclei under consideration can be seen in Table I.

The deformed quasiparticle mean field is now complemented with a spin-isospin separable residual interaction that contains two parts, an attractive particle-hole and a repulsive particle-particle. The coupling strengths of these forces, $\chi_{\mathrm{GT}}^{p h}$ and $\kappa_{\mathrm{GT}}^{p p}$, are defined as positive. The strength of the $p h$ force is usually determined by adjusting the calculated positions of the GT giant resonances to experiment. The strength of the $p p$ force is determined by fitting the $\beta$-decay half-lives of $\beta$ emitters. This fitting procedure was systematically carried out in Ref. [8], where the strengths $\chi_{\mathrm{GT}}^{p h}$ and $\kappa_{\mathrm{GT}}^{p p}$ were considered to be smooth functions of the mass number $A$. The result found using a Nilsson potential as the deformed mean field was $\chi_{\mathrm{GT}}^{p h}=5.2 / A^{0.7} \mathrm{MeV}$ and $\kappa_{\mathrm{GT}}^{p p}=0.58 / A^{0.7} \mathrm{MeV}$. Nevertheless, this parametrization clearly depends on the model used for single-particle wave functions and energies, as well as on the set of experimental data considered. Therefore, these coupling strengths can be used as a reference but cannot be safely extrapolated to different mean fields or different mass regions. As we shall see in the next section, the strengths from Ref. [8] reproduce well the data when using the WS potential, but one needs a somewhat smaller value of $\chi_{\mathrm{GT}}^{p h}$ to reproduce the GT resonance with the HF mean field.

We introduce the proton-neutron QRPA phonon operator for GT excitations in even-even nuclei,

$$
\Gamma_{\omega_{K}}^{+}=\sum_{\pi \nu}\left[X_{\pi \nu}^{\omega_{K}} \alpha_{\nu}^{+} \alpha_{\pi}^{+}+Y_{\pi \nu}^{\omega_{K}} \alpha_{\nu} \alpha_{\pi}\right]
$$

where $\alpha^{+}(\alpha)$ are quasiparticle creation (annihilation) operators, $\omega_{K}$ are the RPA excitation energies, and $X_{\pi \nu}^{\omega_{K}}, Y_{\pi \nu}^{\omega_{K}}$ are the forward and backward amplitudes, respectively. The solution of the QRPA equations can be found solving first a dispersion relation [20], which is of fourth order in the excitation energies $\omega_{K}$.

In the intrinsic frame, the GT transition amplitudes connecting the QRPA ground state $|0\rangle$ to one-phonon states $\left|\omega_{K}\right\rangle$ satisfying 
TABLE I. Pairing gaps for protons and neutrons $\Delta_{p}, \Delta_{n}(\mathrm{MeV})$, and charge radii $r_{c}(\mathrm{fm})$.

\begin{tabular}{ccccccc}
\hline \hline Nucleus & $\Delta_{p}$ & $\Delta_{n}$ & Expt. $r_{c}[24]$ & $r_{c}$ Sk3 & $r_{c}$ SG2 & $r_{c}[25]$ \\
\hline${ }^{48} \mathrm{Ca}$ & 2.18 & 1.68 & $3.4736(8)$ & 3.586 & 3.549 & 3.471 \\
${ }^{48} \mathrm{Ti}$ & 1.90 & 1.56 & 3.592 & 3.628 & 3.597 & 3.571 \\
${ }^{76} \mathrm{Ge}$ & 1.56 & 1.54 & $4.127(8)$ & 4.130 & 4.083 & 4.057 \\
${ }^{76} \mathrm{Se}$ & 1.75 & 1.71 & $4.152(9)$ & $4.170-4.180$ & $4.113-4.143$ & 4.119 \\
${ }^{82} \mathrm{Se}$ & 1.41 & 1.54 & $4.122(8)$ & 4.204 & 4.159 & 4.131 \\
${ }^{82} \mathrm{Kr}$ & 1.72 & 1.64 & $4.1921(11)$ & 4.196 & 4.196 & 4.173 \\
${ }^{96} \mathrm{Zr}$ & 1.53 & 0.84 & $4.3508(12)$ & $4.433-4.443$ & $4.342-4.389$ & 4.376 \\
${ }^{96} \mathrm{Mo}$ & 1.53 & 1.03 & $4.377(10)$ & $4.448-4.457$ & $4.369-4.388$ & 4.381 \\
${ }^{100} \mathrm{Mo}$ & 1.60 & 1.36 & $4.447(10)$ & 4.516 & $4.439-4.466$ & 4.448 \\
${ }^{100} \mathrm{Ru}$ & 1.55 & 1.30 & 4.453 & 4.516 & 4.457 & 4.449 \\
${ }^{116} \mathrm{Cd}$ & 1.47 & 1.37 & 4.625 & $4.703-4.715$ & 4.653 & 4.643 \\
${ }^{116} \mathrm{Sn}$ & 1.77 & 1.20 & 4.625 & $4.709-4.753$ & 4.702 & 4.609 \\
${ }^{128} \mathrm{Te}$ & 1.13 & 1.28 & 4.735 & $4.803-4.805$ & 4.746 & 4.732 \\
${ }^{128} \mathrm{Xe}$ & 1.32 & 1.27 & 4.776 & $4.836-4.839$ & $4.782-4.786$ & 4.778 \\
${ }^{130} \mathrm{Te}$ & 1.06 & 1.18 & 4.742 & $4.812-4.816$ & 4.750 & 4.739 \\
${ }^{130} \mathrm{Xe}$ & 1.31 & 1.25 & 4.783 & $4.845-4.846$ & $4.796-4.801$ & 4.784 \\
${ }^{136} \mathrm{Xe}$ & 0.98 & 1.44 & 4.799 & 4.878 & 4.815 & 4.804 \\
${ }^{136} \mathrm{Ba}$ & 1.27 & 1.03 & 4.833 & 4.902 & 4.847 & 4.837 \\
${ }^{150} \mathrm{Nd}$ & 1.23 & 1.05 & 5.047 & 5.114 & 5.055 & 5.046 \\
${ }^{150} \mathrm{Sm}$ & 1.44 & 1.19 & 5.047 & 5.108 & 5.046 & 5.047 \\
\hline \hline
\end{tabular}

$$
\Gamma_{\omega_{K}}|0\rangle=0 \quad \Gamma_{\omega_{K}}^{+}|0\rangle=\left|\omega_{K}\right\rangle
$$

are given by

$$
\left\langle\omega_{K}\left|\sigma_{K} t^{ \pm}\right| 0\right\rangle=\mp M_{ \pm}^{\omega_{K}},
$$

where

$$
\begin{aligned}
& M_{-}^{\omega_{K}}=\sum_{\pi \nu}\left(q_{\pi \nu} X_{\pi \nu}^{\omega_{K}}+\widetilde{q}_{\pi \nu} Y_{\pi \nu}^{\omega_{K}}\right), \\
& M_{+}^{\omega_{K}}=\sum_{\pi \nu}\left(\widetilde{q}_{\pi \nu} X_{\pi \nu}^{\omega_{K}}+q_{\pi \nu} Y_{\pi \nu}^{\omega_{K}}\right),
\end{aligned}
$$

and

$$
\begin{aligned}
& \tilde{q}_{\pi \nu}=u_{\nu} v_{\pi} \Sigma_{K}^{\nu \pi}, \\
& q_{\pi \nu}=v_{\nu} u_{\pi} \Sigma_{K}^{\nu \pi}, \\
& \Sigma_{K}^{\nu \pi}=\left\langle\nu\left|\sigma_{K}\right| \pi\right\rangle,
\end{aligned}
$$

where $v$ 's are occupation amplitudes $\left(u^{2}=1-v^{2}\right)$.

Finally, the GT strength $B\left(\mathrm{GT}^{ \pm}\right)$in the laboratory system for a transition $I_{i} K_{i}\left(0^{+} 0\right) \rightarrow I_{f} K_{f}\left(1^{+} K\right)$ can be obtained as

$$
B\left(\mathrm{GT}^{ \pm}\right)=\frac{g_{A}^{2}}{4 \pi}\left[\delta_{K, 0}\left\langle\omega_{K}\left|\sigma_{0} t^{ \pm}\right| 0\right\rangle^{2}+2 \delta_{K, 1}\left\langle\omega_{K}\left|\sigma_{1} t^{ \pm}\right| 0\right\rangle^{2}\right] .
$$

The $2 \nu \beta \beta$ decay is described in second-order perturbation of the weak interaction as two successive Gamow-Teller transitions via virtual intermediate $1^{+}$states. The half-life of the $2 \nu \beta \beta$ decay

$$
T_{1 / 2}^{2 \nu}\left(0_{\mathrm{gs}}^{+} \rightarrow 0_{\mathrm{gs}}^{+}\right)=\left[G^{2 \nu}\left|M_{\mathrm{GT}}^{2 \nu}\right|^{2}\right]^{-1}
$$

is given as a product of a phase-space integral $G^{2 v}$ and the Gamow-Teller transition matrix element $M_{\mathrm{GT}}^{2 \nu}$, which contains the nuclear structure effects. For a transition connecting initial and final ground states, it is given by

$$
M_{\mathrm{GT}}^{2 \nu}=\sum_{K} \sum_{m_{i}, m_{f}} \frac{\left\langle 0_{f}^{+}\left\|\sigma_{K} t^{-}\right\| \omega_{K}^{m_{f}}\right\rangle\left\langle\omega_{K}^{m_{f}} \mid \omega_{K}^{m_{i}}\right\rangle\left\langle\omega_{K}^{m_{i}}\left\|\sigma_{K} t^{-}\right\| 0_{i}^{+}\right\rangle}{\left(\omega_{K}^{m_{f}}+\omega_{K}^{m_{i}}\right) / 2}
$$

where $K=0, \pm 1$ and $m_{i}, m_{f}$ label the number of intermediate $1^{+}$RPA states $\omega_{K}^{m_{i}}, \omega_{K}^{m_{f}}$ reached from the initial $\left|0_{i}^{+}\right\rangle$and final $\left|0_{f}^{+}\right\rangle$nuclear ground states, respectively. The overlap $\left\langle\omega_{K}^{m_{f}} \mid \omega_{K}^{m_{i}}\right\rangle$ is needed to take into account the nonorthogonality of the intermediate states reached from the initial ground state to those reached from the final ground state. It is given by [12]

$$
\left\langle\omega_{K}^{m_{f}} \mid \omega_{K}^{m_{i}}\right\rangle=\sum_{\ell_{i} \ell_{f}}\left[X_{\ell_{f}}^{\omega_{K}} X_{\ell_{i}}^{\omega_{K}}-Y_{\ell_{f}}^{\omega_{K}^{m_{f}}} Y_{\ell_{i}}^{\omega_{K}}\right] R_{\ell_{f} \ell_{i}}\left\langle B C S_{f} \mid B C S_{i}\right\rangle,
$$

where $\ell_{i} \ell_{f}$ label the quasiparticle $\pi \nu$ pairs for the initial and final nucleus, respectively. The factor $R_{\ell_{f} \ell_{i}}$ includes the overlap of single-particle wave functions of the initial and final nuclei [12], 


$$
R_{\ell \ell^{\prime}}=\left\langle\pi \mid \pi^{\prime}\right\rangle\left\langle\nu \mid \nu^{\prime}\right\rangle\left(u_{\pi}^{i} u_{\pi^{\prime}}^{f}+v_{\pi}^{i} v_{\pi^{\prime}}^{f}\right)\left(u_{\nu^{\prime}}^{i} u_{\nu^{\prime}}^{f}+v_{\nu}^{i} v_{\nu^{\prime}}^{f}\right) .
$$

The BCS overlap factor $\left\langle B C S_{f} \mid B C S_{i}\right\rangle$ is derived in Ref. [12]. An approximate expression is given by

$$
\left\langle B C S_{f} \mid B C S_{i}\right\rangle \approx \prod_{\Omega_{\pi}} \prod_{k=1}^{N_{\Omega_{\pi}}}\left(u_{k}^{f} u_{k}^{i}+v_{k}^{f} v_{k}^{i}\right) \prod_{\Omega_{\nu}}^{N_{\Omega_{v}}} \prod_{j=1}\left(u_{j}^{f} u_{j}^{i}+v_{j}^{f} v_{j}^{i}\right),
$$

with $N_{\Omega}$ the number of single-particle states with the same values of parity and projection $\Omega$ of the full angular momentum on the nuclear symmetry axis.

We note that for the case of the spherical QRPA, the derivation of the overlap factor of the intermediate nuclear states generated from the initial and final nuclei was outlined in Ref. [21]. For the case of the deformed QRPA, the generalization of this derivation was presented in [12]. There, the importance of the BCS overlap factor, which is an integral part of the overlap factor of the two sets of the intermediate nucleus, on the evaluation of the double $\beta$ decay nuclear matrix elements was maintained. Then, this procedure of the derivation of the overlap factor of intermediate nuclear states was followed also in Ref. [15], however with a significant approximation. The role of the BCS overlap factor was neglected. Of course, this can affect the final results significantly, especially in the cases when deformations of the initial and final nuclei are different.

\section{GROUND-STATE PROPERTIES}

In this section, we present results for the bulk properties of the nuclei under study based on the quasiparticle meanfield description. We consider both the WS potential and Skyrme HF approaches.

In the case of HF, the first step is to study the energy surfaces as a function of deformation. For this purpose, we perform constrained calculations [22], minimizing the HF energy under the constraint of keeping fixed the nuclear deformation. We can see in Fig. 1 the total HF energy plotted versus the quadrupole deformation parameter

$$
\beta=\sqrt{\frac{\pi}{5}} \frac{Q_{p}}{Z r_{c}^{2}},
$$

defined in terms of the microscopically calculated quadrupole moment $Q_{p}$ and charge root mean-square radius $r_{c}$.

The results in Fig. 1 correspond to HF calculations with the force Sk3, which are qualitatively similar to the results obtained with other Skyrme forces, such as SG2 and SLy4 [23]. We observe that the HF calculation predicts in some instances ( $\mathrm{Ge}, \mathrm{Se}, \mathrm{Zr}, \mathrm{Cd}, \mathrm{Sn}, \mathrm{Te}, \mathrm{Xe})$ the existence of two energy minima close in energy, giving rise to possible shape isomers in these nuclei. Solid lines represent the energy curves of the parent nuclei suffering the double $\beta$ decay, while dashed lines represent the energy curves of the corresponding daughter nuclei.

We can see in Table I the experimental and the microscopically calculated charge root mean-square radii $r_{c}$ with

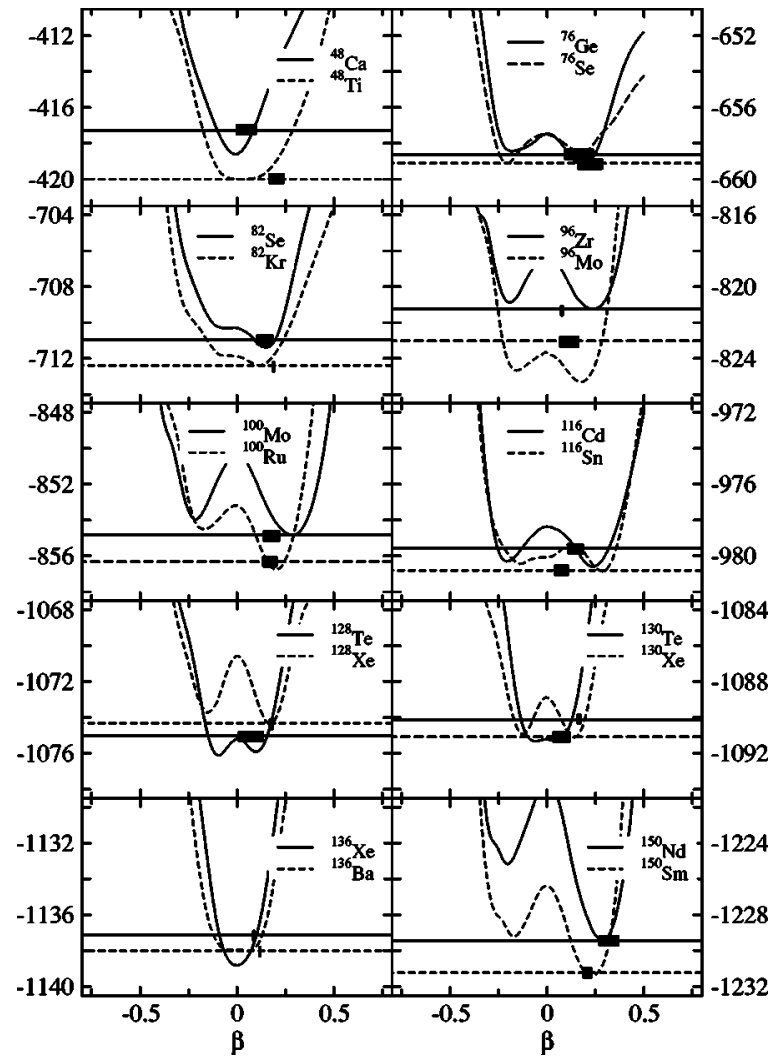

FIG. 1. Binding energy $(\mathrm{MeV})$ as a function of the quadrupole deformation parameter $\beta$ obtained from deformed Hartree-Fock calculations with the Skyrme force Sk3. Experimental $\beta$ values from Refs. [26] and [27] are represented as the extreme values of the black boxes. The experimental difference of binding parent and daughter binding energies is given by the distance between the two horizontal lines (see text).

the forces Sk3 and SG2. We quote the two, oblate-prolate, results in those cases where the energies for the two shapes are very close. The values obtained for the charge radii are in good agreement with the experimental values from Ref. [24]. They are also in good agreement with the results obtained from relativistic mean-field calculations [25].

In Table II, we can see the theoretical and experimental quadrupole deformation. Experimental values have been extracted from the measured quadrupole moments from two different methods. In the first one, the quadrupole deformation is obtained from Eq. (3.1), using the empirical intrinsic moments derived from the laboratory moments of Ref. [26] assuming a well defined deformation. In the second case, the quadrupole deformations are taken from Ref. [27], where they were derived from experimental values of $B(E 2)$ strengths. In this case, the sign cannot be extracted.

Our theoretical values have been derived microscopically from the forces Sk3 and SG2, using the intrinsic quadrupole moments obtained as ground-state expectations of the $Q_{20}$ operator and the microscopic charge radii quoted in Table I. We show the results obtained for the equilibrium shapes using the Skyrme forces Sk3 and SG2. In those cases where a second minimum appears at close energy, we also show within square brackets the corresponding deformation. We 
TABLE II. Theoretical and experimental $\beta$ values, see text.

\begin{tabular}{|c|c|c|c|c|c|c|}
\hline \multirow[b]{2}{*}{ Nucleus } & \multicolumn{2}{|c|}{ Expt. } & \multicolumn{4}{|c|}{ Theory } \\
\hline & Ref. [26] & Ref. [27] & This work (Sk3) & This work (SG2) & Ref. [25] & Ref. [28] \\
\hline${ }^{48} \mathrm{Ca}$ & 0.000 & $0.101(17)$ & -0.002 & -0.001 & 0.000 & 0.000 \\
\hline${ }^{48} \mathrm{Ti}$ & $+0.17(10)$ & $0.269(7)$ & -0.002 & -0.003 & -0.009 & 0.000 \\
\hline${ }^{76} \mathrm{Ge}$ & $+0.095(30)$ & $0.2623(39)$ & 0.161 & 0.157 & 0.157 & 0.143 \\
\hline${ }^{76} \mathrm{Se}$ & $+0.163(33)$ & $0.3090(37)$ & $-0.181[+0.157]$ & $-0.191[+0.049]$ & -0.244 & -0.241 \\
\hline${ }^{82} \mathrm{Se}$ & $+0.104(32)$ & $0.1944(26)$ & 0.126 & 0.150 & 0.133 & 0.154 \\
\hline${ }^{82} \mathrm{Kr}$ & & $0.2022(45)$ & 0.106 & 0.103 & 0.119 & 0.071 \\
\hline${ }^{96} \mathrm{Zr}$ & & $0.081(16)$ & $0.207[-0.167]$ & $0.016[+0.147]$ & 0.223 & 0.217 \\
\hline${ }^{96} \mathrm{Mo}$ & $+0.068(27)$ & $0.1720(16)$ & $0.147[-0.164]$ & $-0.006[+0.119]$ & 0.167 & 0.080 \\
\hline${ }^{100} \mathrm{Mo}$ & $+0.139(30)$ & $0.2309(22)$ & 0.236 & $0.167[-0.191]$ & 0.253 & 0.244 \\
\hline${ }^{100} \mathrm{Ru}$ & $+0.136(22)$ & $0.2172(22)$ & 0.175 & 0.157 & 0.194 & 0.161 \\
\hline${ }^{116} \mathrm{Cd}$ & $+0.113(11)$ & $0.1907(34)$ & $0.206[-0.207]$ & 0.209 & -0.258 & -0.241 \\
\hline${ }^{116} \mathrm{Sn}$ & $+0.043(10)$ & $0.1118(16)$ & $0.264[-0.134]$ & $0.251[-0.034]$ & 0.003 & 0.000 \\
\hline${ }^{128} \mathrm{Te}$ & $+0.011(10)$ & $0.1363(11)$ & $-0.088[+0.102]$ & $0.094[-0.091]$ & -0.002 & 0.000 \\
\hline${ }^{128} \mathrm{Xe}$ & & $0.1837(49)$ & $0.148[-0.122]$ & $0.150[-0.133]$ & 0.160 & 0.143 \\
\hline${ }^{130} \mathrm{Te}$ & $+0.035(23)$ & $0.1184(14)$ & $-0.076[+0.051]$ & $-0.039[+0.066]$ & 0.032 & 0.000 \\
\hline${ }^{130} \mathrm{Xe}$ & & $0.169(6)$ & $0.108[-0.098]$ & $0.161[-0.132]$ & 0.128 & -0.113 \\
\hline${ }^{136} \mathrm{Xe}$ & & $0.086(19)$ & 0.001 & 0.016 & -0.001 & 0.000 \\
\hline${ }^{136} \mathrm{Ba}$ & & $0.1242(8)$ & 0.009 & 0.070 & -0.002 & 0.000 \\
\hline${ }^{150} \mathrm{Nd}$ & $+0.367(86)$ & $0.2848(21)$ & 0.266 & 0.271 & 0.221 & 0.243 \\
\hline${ }^{150} \mathrm{Sm}$ & $+0.230(30)$ & $0.1931(22)$ & 0.207 & 0.203 & 0.176 & 0.206 \\
\hline
\end{tabular}

compare our results with the results from relativistic meanfield calculations of Ref. [25] and with results from systematic calculations [28] based on macroscopic-microscopic models (finite range droplet macroscopic model and folded Yukawa single-particle microscopic model). The agreement between all the theoretical calculations is very remarkable and in general they are within the range of experimental values determined from Refs. [26] and [27]. The experimental $\beta$ values from Refs. [26] and [27] are represented in Fig. 1 by the end points of the black boxes.

There is still another important piece of experimental information relevant to double $\beta$ decay that we wish to explore here. This is the $Q_{\beta \beta}$ energy of the decay. The energy released in a double $\beta$ process in a transition from ground-state to ground-state is given by

$$
\begin{aligned}
Q_{\beta \beta} & =\left[M_{\text {parent }}-M_{\text {daughter }}-2 m_{e}\right] \\
& =\left[2\left(m_{n}-m_{p}-m_{e}\right)+B E(Z, N)-B E(Z+2, N-2)\right],
\end{aligned}
$$

in terms of the nuclear masses $M$ 's, or similarly, in terms of the binding energies $B E$ 's of parent $(Z, N)$ and daughter $(Z+2, N-2)$ nuclei.

We can see in Table III the experimental values of $Q_{\beta \beta}$. They are compared with the values calculated with the force Sk3. The agreement with experiment is reasonable taking into account that we are dealing with differences of energies ranging from $400 \mathrm{MeV}$ in $A=48$ systems to $1200 \mathrm{MeV}$ in $A=150$ and a tiny percent error in the theoretical masses may induce a relatively large error in $Q_{\beta \beta}$ values. From Table III, we can see that to match the experimental energies, we need to increase slightly the energy difference in $A=48,76,82,116,128,130,136$ and to decrease it in $A=96,100,150$.

In this work, we have used Sk3 as a representative of the Skyrme forces without any attempt to optimize the agreement with the experimental $Q_{\beta \beta}$ values. Even if Sk3 force may not be the best Skyrme force to accurately predict the right $Q_{\beta \beta}$ values, it is very interesting to see that experimental and theoretical values are in many cases quite close. For the cases examined here, it appears that the $Q_{\beta \beta}$ values are

TABLE III. Experimental and theoretical $Q_{\beta \beta}(\mathrm{MeV})$ values obtained with the Skyrme force Sk3.

\begin{tabular}{crr}
\hline \hline Double $\beta$ transition & $\left(Q_{\beta \beta}\right)_{\text {exp }}$ & $\left(Q_{\beta \beta}\right)_{\mathrm{Sk} 3}$ \\
\hline${ }^{48} \mathrm{Ca} \rightarrow{ }^{48} \mathrm{Ti}$ & 4.272 & 2.95 \\
${ }^{76} \mathrm{Ge} \rightarrow{ }^{76} \mathrm{Se}$ & 2.039 & 1.36 \\
${ }^{82} \mathrm{Se} \rightarrow{ }^{82} \mathrm{Kr}$ & 2.995 & 2.58 \\
${ }^{96} \mathrm{Zr} \rightarrow{ }^{96} \mathrm{Mo}$ & 3.350 & 5.59 \\
${ }^{100} \mathrm{Mo} \rightarrow{ }^{100} \mathrm{Ru}$ & 3.034 & 3.57 \\
${ }^{116} \mathrm{Cd} \rightarrow{ }^{116} \mathrm{Sn}$ & 2.805 & 1.88 \\
${ }^{128} \mathrm{Te} \rightarrow{ }^{128} \mathrm{Xe}$ & 0.867 & -0.10 \\
${ }^{130} \mathrm{Te} \rightarrow{ }^{130} \mathrm{Xe}$ & 2.529 & 1.20 \\
${ }^{136} \mathrm{Xe} \rightarrow{ }^{136} \mathrm{Ba}$ & 2.468 & 0.80 \\
${ }^{150} \mathrm{Nd} \rightarrow{ }^{150} \mathrm{Sm}$ & 3.367 & 3.59 \\
\hline \hline
\end{tabular}



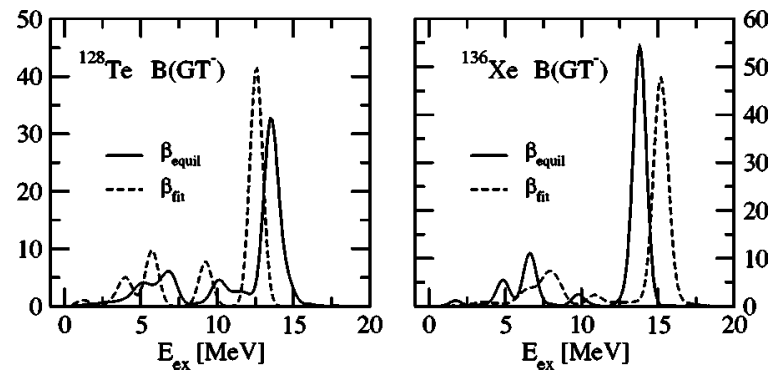

FIG. 2. HF-Sk3 $B\left(\mathrm{GT}^{-}\right)$strength distributions $\left[g_{A}^{2} / 4 \pi\right]$ in ${ }^{128} \mathrm{Te}$ and ${ }^{136} \mathrm{Xe}$ calculated with the equilibrium deformation $\left(\beta_{\text {equil }}\right)$ and with the deformation that fits the experimental $Q_{\beta \beta}$ values $\left(\beta_{\mathrm{fit}}\right)$.

fairly well reproduced for $A=82,100,150$ and that deviations from experiment in the worst case are $2.25 \mathrm{MeV}$ in $A=96$, where $Q_{\beta \beta(\exp )}=3.35 \mathrm{MeV}$ and $Q_{\beta \beta(\mathrm{Sk} 3)}=5.59 \mathrm{MeV}$. In the future, it will be interesting to test other Skyrme forces which may give better fits to the $Q_{\beta \beta}$ values. A case of particular concern for double $\beta$ decay calculations is that of $A$ $=128$ for which the Sk3 force gives $Q_{\beta \beta}=-0.10 \mathrm{MeV}$. Clearly, in this case further work is needed to make selfconsistent calculations that give the right $Q_{\beta \beta}$ and GT strengths.

We also notice that, as illustrated in Fig. 1, by changing slightly the deformation value in the vicinity of the equilibrium deformation, one can change the binding energy correspondingly and get into agreement with the experimental $Q_{\beta \beta}$ value. This procedure could be justified from the point of view that, in principle, in the HF method one could consider several collective degrees of freedom and that the absolute minimum in the multidimensional landscape could correspond to a slightly different $\beta$ value. We can see in Fig. 1 the experimental value of $\delta=B E_{i}-B E_{f}$ as the distance between the two horizontal lines plotted in each panel. The solid horizontal line refers to the energy of the parent while the dashed horizontal one refers to the daughter binding energy. One of them is always a reference and signals the energy to keep fixed (parent for $A=96,100,150$ and daughter in $A=48,76,82,116,128,130,136)$. The other line indicates the binding energy needed to reproduce the experimental $\delta$ $=B E_{i}-B E_{f}$. Therefore, the cuts of this horizontal line with the corresponding energy curve indicates the deformations where this condition is satisfied.

Figure 2 shows the results obtained with HF-Sk3 for the GT strength distributions in ${ }^{128} \mathrm{Te}$ and ${ }^{136} \mathrm{Xe}$, which are among the cases where the calculated $Q_{\beta \beta}$ is worse and the change in $\beta$ needed to fit $Q_{\beta \beta}$ is larger. We show the results obtained with the equilibrium deformations $\beta=-0.088$ in ${ }^{128} \mathrm{Te}$ and $\beta=0.001$ in ${ }^{136} \mathrm{Xe}$ as well as with the deformations that fit the $Q_{\beta \beta}$ values, $\beta=-0.005$ in ${ }^{128} \mathrm{Te}$ and $\beta=0.102$ in ${ }^{136} \mathrm{Xe}$. We can see that the strength distributions obtained with both deformations are similar except for a small displacement in energies. In all the other cases the effect is even smaller and the strength distributions obtained with the equilibrium deformation or with the slightly changed deformation are practically unchanged. In the next section, we show GT strengths obtained at the HF minimum.

In the case of the Woods-Saxon potential, where the deformation is an input parameter, we take the values from
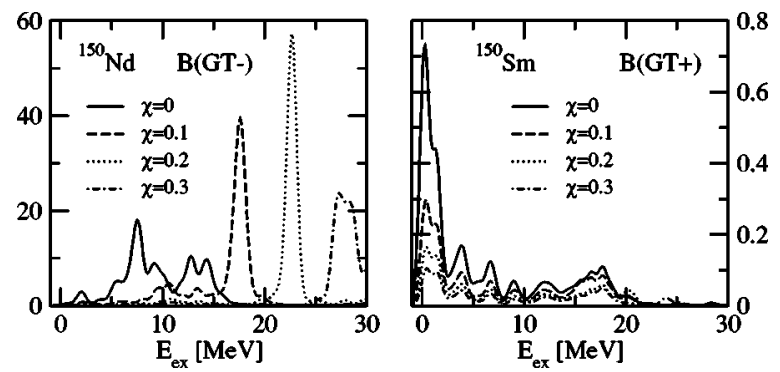

FIG. 3. HF-Sk3 Gamow-Teller strength distributions $\left[g_{A}^{2} / 4 \pi\right]$ in ${ }^{150} \mathrm{Nd}$ and ${ }^{150} \mathrm{Sm}$ for various values of the coupling strength $\chi_{\mathrm{GT}}^{p h}(\mathrm{MeV})$.

both Refs. [26] and [27]. Since for each nucleus the two references give two different values of the $\beta$ parameter, we show in the next section the GT distributions obtained with the two values to take into account this uncertainty.

\section{GAMOW-TELLER STRENGTH DISTRIBUTIONS}

In this section, we show and discuss the Gamow-Teller strength distributions obtained from different choices of the deformed mean fields and residual interactions.

We notice that the relevant strength distributions for the double $\beta$ decay are the $B\left(\mathrm{GT}^{-}\right)$distribution of the parent nuclei and the $B\left(\mathrm{GT}^{+}\right)$distribution of daughter nuclei.

As a general rule, the following figures showing the GT strength distributions are plotted versus the excitation energy of the daughter nucleus. The distributions of the GT strength have been folded with Gaussian functions of $1 \mathrm{MeV}$ width to facilitate the comparison among the various calculations, so that the original discrete spectrum is transformed into a continuous profile. These distributions are given in units of $g_{A}^{2} / 4 \pi$ and one should keep in mind that a quenching of the $g_{A}$ factor, typically $g_{A, \text { eff }}=(0.7-0.8) g_{A \text {,free, which appears }}$ squared in the GT strength, is expected on the basis of the observed quenching in charge exchange reactions.

In the case of the $B\left(\mathrm{GT}^{+}\right)$distributions, we first observe the different scale, which is about one order of magnitude smaller than the $B\left(\mathrm{GT}^{-}\right)$scale. This is a consequence of the Pauli blocking. In the nuclei considered here, the number of neutrons $N$ is much larger than the number of protons $Z$. The difference between total $B\left(\mathrm{GT}^{-}\right)$and $B\left(\mathrm{GT}^{+}\right)$strengths (Ikeda sum rule, which is fulfilled in our calculations) is then
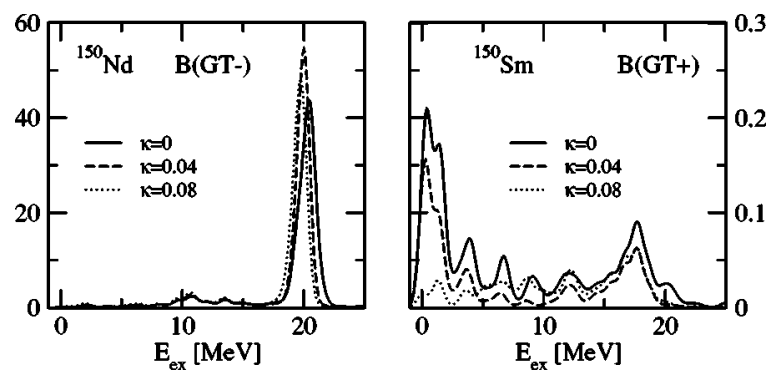

FIG. 4. HF-Sk3 Gamow-Teller strength distributions $\left[g_{A}^{2} / 4 \pi\right]$ in ${ }^{150} \mathrm{Nd}$ and ${ }^{150} \mathrm{Sm}$ for various values of the coupling strength $\kappa_{\mathrm{GT}}^{p p}(\mathrm{MeV})$. 


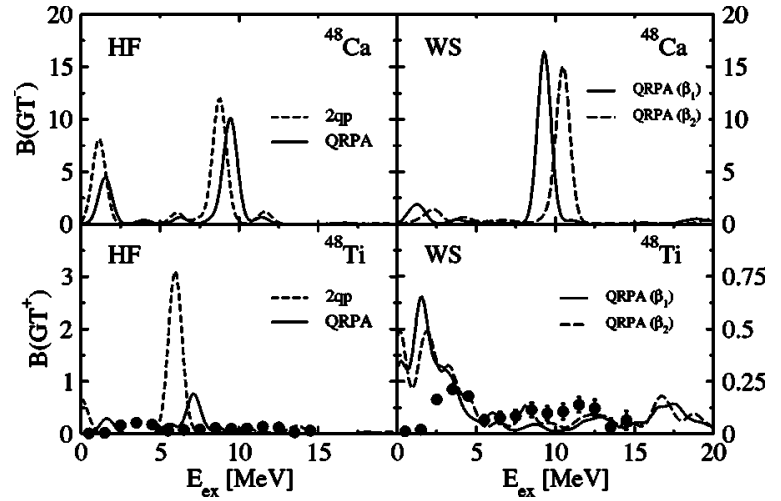

FIG. 5. Gamow-Teller $B\left(\mathrm{GT}^{-}\right)$and $B\left(\mathrm{GT}^{+}\right)$strength distributions $\left[g_{A}^{2} / 4 \pi\right]$ in ${ }^{48} \mathrm{Ca}$ and ${ }^{48} \mathrm{Ti}$ plotted as a function of the excitation energies of the corresponding daughter nuclei. Left panels show results from $\mathrm{HF}(\mathrm{Sk} 3)$ calculations without residual interaction (dashed lines) and with residual interactions with $\chi_{\mathrm{GT}}^{p h}=0.10 \mathrm{MeV}$, $\kappa_{\mathrm{GT}}^{p p}=6 / \mathrm{A} \mathrm{MeV}$ (solid lines). Right panels show results using WS potentials with $\chi_{\mathrm{GT}}^{p h}$ and $\kappa_{\mathrm{GT}}^{p p}$ from Ref. [8] and with two different values for the quadrupole deformation: $\beta_{1}$ from Ref. [26] (solid line) and $\beta_{2}$ from Ref. [27] (dashed line). Experimental data are from Ref. [30]. Notice that no quenching factor has been included in the calculations.

a large number given by $3(N-Z)$ and practically determined by the magnitude of the $B\left(\mathrm{GT}^{-}\right)$strength.

We start in Fig. 3 with a discussion of the dependence of the GT strength distributions on the coupling strength of the particle-hole residual interaction $\chi_{\mathrm{GT}}^{p h}$ for a fixed value of the particle-particle coupling constant $\kappa_{\mathrm{GT}}^{p p}=0$. The results correspond to HF with the force Sk3 in the $A=150$ case. We can see on the left panel the $B\left(\mathrm{GT}^{-}\right)$strength distribution of the parent nucleus ${ }^{150} \mathrm{Nd}$ and on the right panel the $B\left(\mathrm{GT}^{+}\right)$ strength distribution of the daughter nucleus ${ }^{150} \mathrm{Sm}$. The pairing gap parameters are given in Table I and the deformations are given in Table II. We can see in Fig. 3 how the most important effect of $\chi_{\mathrm{GT}}^{p h}$ on the $B\left(\mathrm{GT}^{-}\right)$strength distribution is a shift of the strength toward higher excitation energies. This displacement of the GT strength is accompanied by a reduction of the strength. This reduction can be more clearly appreciated on the $B\left(\mathrm{GT}^{+}\right)$strength distribution because the scale in this case is about two orders of magnitude smaller,

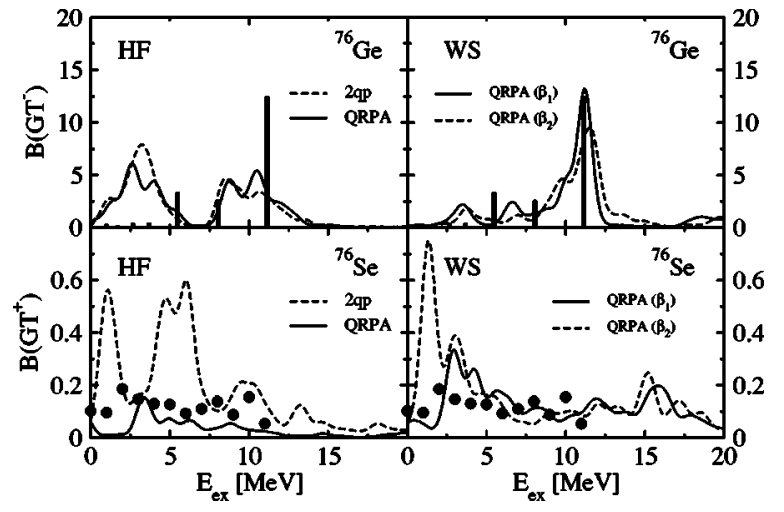

FIG. 6. Same as in Fig. 5 for ${ }^{76} \mathrm{Ge}$ and ${ }^{76} \mathrm{Se}$. Data in ${ }^{76} \mathrm{Se}$ are from [31]. Vertical lines in ${ }^{76} \mathrm{Ge}$ are experimental data from [32].

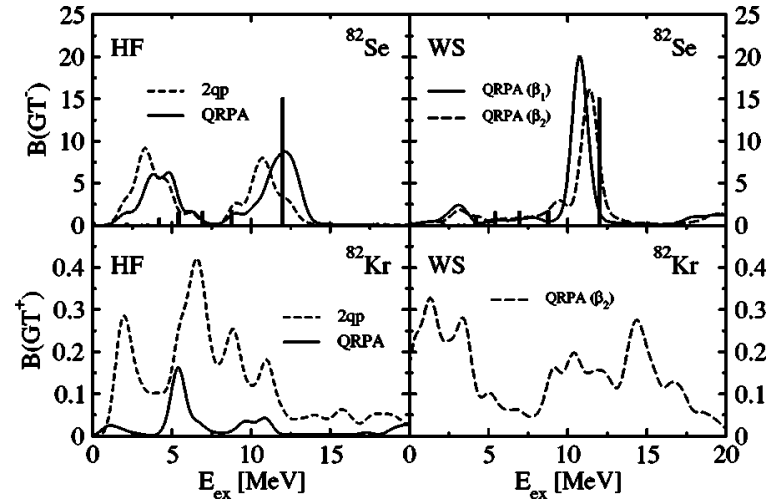

FIG. 7. Same as in Fig. 5 for ${ }^{82} \mathrm{Se}$ and ${ }^{82} \mathrm{Kr}$. Vertical lines in ${ }^{82} \mathrm{Se}$ are experimental data from [32].

as it should be to fulfill the Ikeda sum rule $\Sigma\left[B\left(\mathrm{GT}^{-}\right)\right.$ $\left.-B\left(\mathrm{GT}^{+}\right)\right]=3(N-Z)=90,78$ for $\mathrm{Nd}$ and $\mathrm{Sm}$, respectively. Therefore, the coupling constant $\chi_{\mathrm{GT}}^{p h}$ plays an important role to reproduce the position of the $\mathrm{GT}^{-}$resonance. On the other hand, the sensitivity of the GT strength distribution on the particle-particle coupling constant $\kappa_{\mathrm{GT}}^{p p}$ is not so important, as can be seen from Fig. 4, where we can see the GT strength distributions for a fixed value of $\chi_{\mathrm{GT}}^{p h}=0.156 \mathrm{MeV}$ [8] and for several values of $\kappa_{\mathrm{GT}}^{p p}$ on the example of HF with the force Sk3 in the $A=150$ case. As we can see, the position of the resonance does not change appreciably. Therefore, other methods, such as fitting the half-lives of unstable nuclei in the same mass region, have to be used to get phenomenologically their values.

In the next set of figures (Figs. 5-14) we show, for each couple of double $\beta$ decay partners, the results obtained for the $B\left(\mathrm{GT}^{-}\right)$strength distributions of the parent nuclei on the top panels and for the $B\left(\mathrm{GT}^{+}\right)$strength distributions of the daughter nuclei on the bottom panels. Also shown are the experimental data whenever they are available. In each figure, the left panels correspond to $\mathrm{HF}+\mathrm{BCS}+\mathrm{QRPA}$ calculations with the force Sk3 and the right panels to WS+BCS + QRPA calculations. In the case of HF, we use the equilibrium deformations. We show with dashed lines the 2qp results for $\mathrm{HF}+\mathrm{BCS}$ calculations where the residual interaction is not considered. This serves as a reference and can be used to see the necessity of the residual force to get into agree-

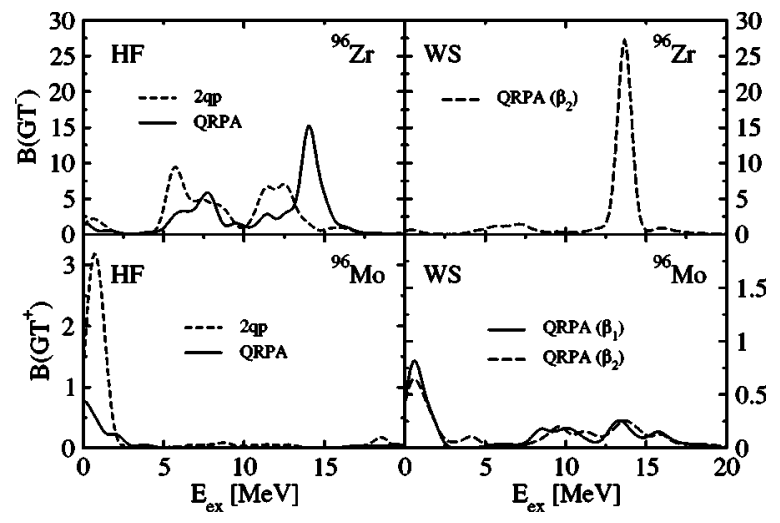

FIG. 8. Same as in Fig. 5 for ${ }^{96} \mathrm{Zr}$ and ${ }^{96} \mathrm{Mo}$. 


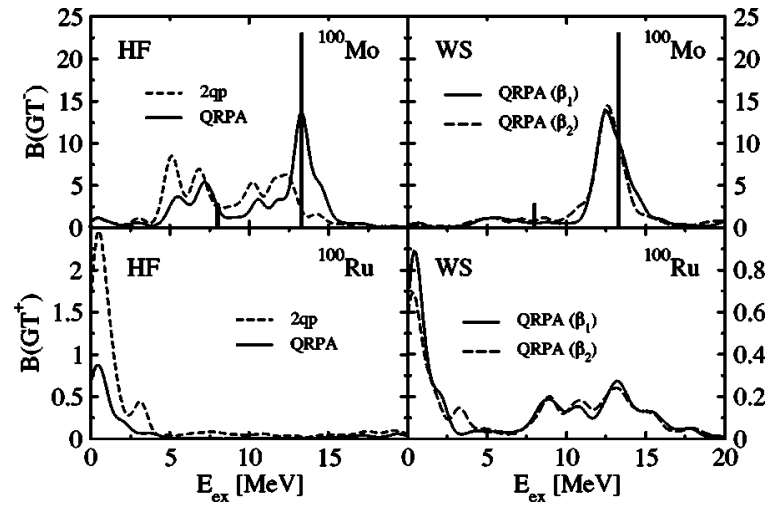

FIG. 9. Same as in Fig. 5 for ${ }^{100} \mathrm{Mo}$ and ${ }^{100} \mathrm{Ru}$. Vertical lines in ${ }^{100}$ Mo are experimental data from [33].

ment with experiment. Solid lines are the results obtained with $\chi_{\mathrm{GT}}^{p h}=0.1 \mathrm{MeV}$ and $\kappa_{\mathrm{GT}}^{p p}=6 / A \mathrm{MeV}$, which produce a good fit to all the measured GT resonances of double $\beta$ emitters, as well as to the two-neutrino double $\beta$ decay matrix elements, as we shall see in the next section. This small value of the $\chi_{\mathrm{GT}}^{p h}$ coupling constant needed to reproduce the experimental GT resonances within a self-consistent approach with Skyrme forces is in agreement with the same observation reported in Refs. [11,13] and reflects the fact that one needs less residual interaction when using realistic effective density-dependent forces than when using phenomenological potentials to generate the single-particle energies and wave functions.

In the case of calculations with the WS potentials shown on the right-hand panels, we show results for the two different experimental deformations as obtained from Refs. [26] (solid lines) and [27] (dashed lines), which are given in Table II. The calculations are done for a fixed value of the $\chi_{\mathrm{GT}}^{p h}$ and $\kappa_{\mathrm{GT}}^{p p}$ constants as obtained from the parametrization in Ref. [8].

Some general common features to all figures can be established first. Concerning the HF calculations, the value of $\chi_{\mathrm{GT}}^{p h}$ given by the parametrization of Ref. [8] is an overestimation when dealing with self-consistent Skyrme HF calculations. Actually, a small value of $\chi_{\mathrm{GT}}^{p h}=0.1 \mathrm{MeV}$ is already able to reproduce the experimental position of the GT reso-

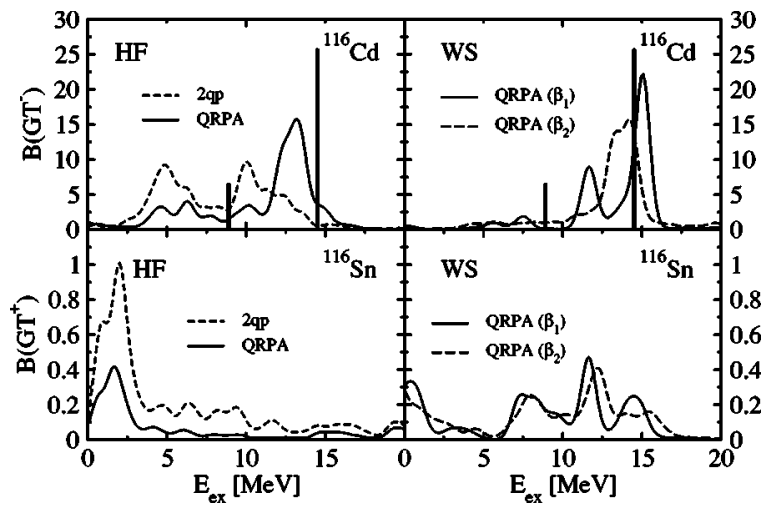

FIG. 10. Same as in Fig. 5 for ${ }^{116} \mathrm{Cd}$ and ${ }^{116} \mathrm{Sn}$. Vertical lines in ${ }^{116} \mathrm{Cd}$ are experimental data from [33].

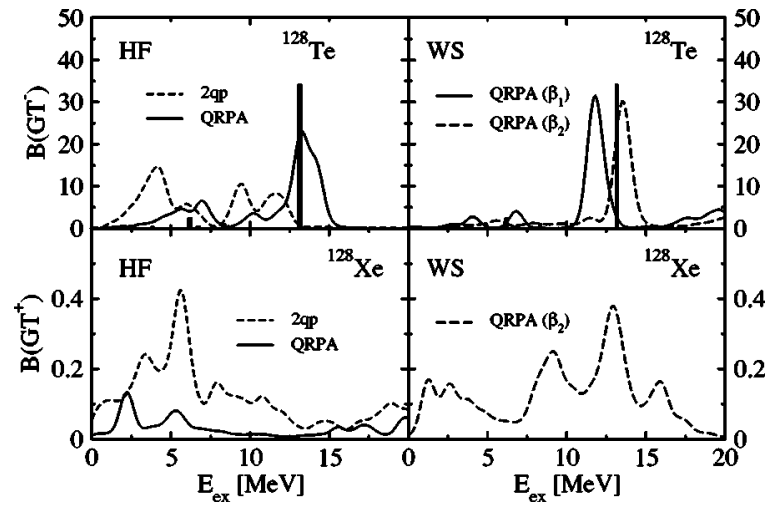

FIG. 11. Same as in Fig. 5 for ${ }^{128}$ Te and ${ }^{128} \mathrm{Xe}$. Vertical lines in ${ }^{128} \mathrm{Te}$ are experimental data from [32].

nance. This is a consequence of the structure of the two-body density-dependent Skyrme force that contains terms like spin exchange operators leading to a spin-spin interaction in the self-consistent mean field, which is absent in the WS potential. The agreement with the experimental energy of the GT resonance is in this case very good, as can be seen in the cases $A=76,82,100,116,128,130$, where this information is available. Indeed, the experimental giant GT resonances shown in these figures represent the centroids of broad bumps. The resonance in ${ }^{48} \mathrm{Ca}$ reported at $10 \mathrm{MeV}$ in Ref. [29] and used in the fitting procedure of Ref. [8] is also in good agreement with our results.

With respect to the calculations performed with the WS potential, we can see that larger deformations produce peaks in the GT distributions displaced to higher energies. This is a consequence of the larger separation of the single-particle energies when the deformation increases. Thus, since the deformation derived from Ref. [27] is larger than that of Ref. [26], solid lines appear in general on the left of dashed lines.

It is also remarkable the good agreement with experiment obtained in this case. This agreement is mainly determined by the fixed value of $\chi_{\mathrm{GT}}^{p h}$ from Ref. [8], which is still valid when describing the mean field with a WS potential. One should keep in mind that the parametrization of Ref. [8] was obtained using a Nilsson potential.

In Table IV, we compare the total GT strength measured and calculated with both HF and WS. When a standard

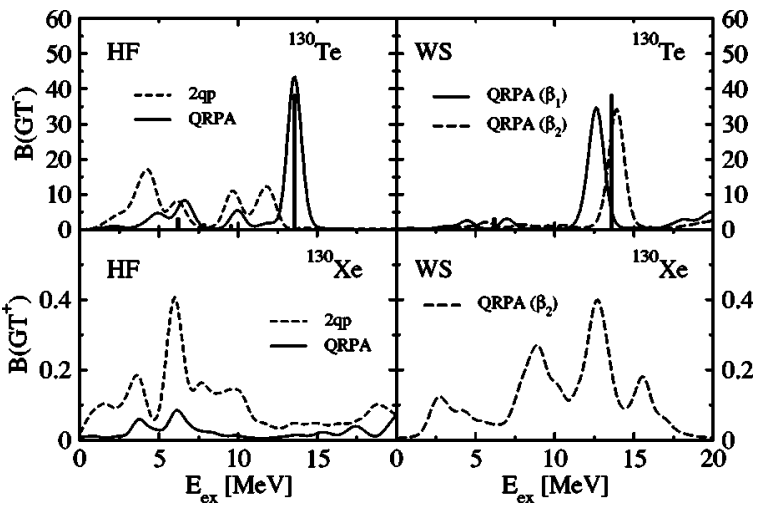

FIG. 12. Same as in Fig. 5 for ${ }^{130}$ Te and ${ }^{130}$ Xe. Vertical lines in ${ }^{130} \mathrm{Te}$ are experimental data from [32]. 


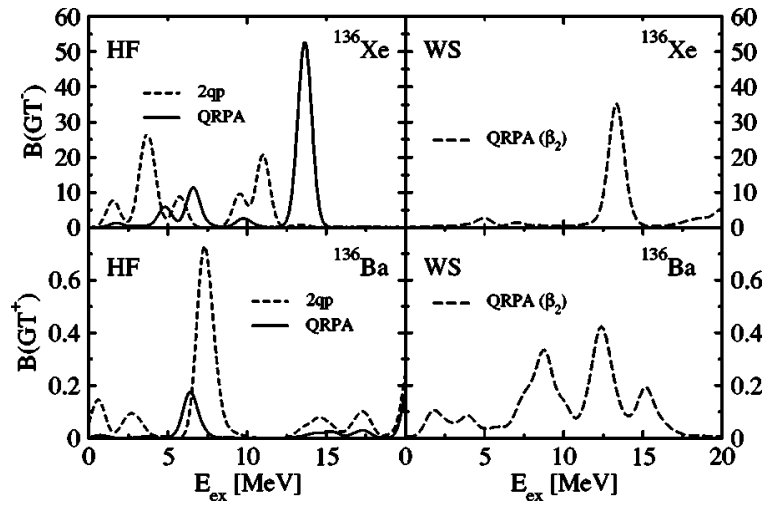

FIG. 13. Same as in Fig. 5 for ${ }^{136} \mathrm{Xe}$ and ${ }^{136} \mathrm{Ba}$.

quenching factor of 0.6 is included in the theoretical results, fair agreement is found between theory and experiment.

\section{TWO-NEUTRINO DOUBLE $\beta$ DECAY MATRIX ELEMENTS}

In this section, we analyze the effects of deformation, as well as the effect of the mean field and residual interactions on the $2 \nu \beta \beta$-decay. First, we discuss the sensitivity to deformation of the nuclear structure contribution $M_{\mathrm{GT}}^{2 \nu}$ to the $2 \nu \beta \beta$ half-lives. This is done in Fig. 15, where we show the matrix elements $M_{\mathrm{GT}}^{2 \nu}$ as a function of both parent and daughter deformations. The figure corresponds to the decay ${ }^{96} \mathrm{Zr}$ $\rightarrow{ }^{96} \mathrm{Mo}$ calculated within a Woods-Saxon scheme with residual interactions from Ref. [8]. We have changed freely the deformations of both parent and daughter nuclei without any constraint from experiment. In this way, we can study qualitatively the effect of deformation. The experimental values for $M_{\mathrm{GT}}^{2 \nu}$ shown in Fig. 15, as well as in the next figures, have been extracted from the adopted experimental half-lives $T_{1 / 2}^{2 \nu}$ given in Ref. [34]. From the experimental half-lives and the corresponding kinematical factors $G^{2 \nu}$, we extract two experimental nuclear matrix elements from Eq. (2.7) by assuming values for the axial coupling constant $g_{A}=1.25$ or $g_{A}=1$. These two values are plotted in Figs. 15-18 as horizontal lines.

From Fig. 15 we can see that the matrix elements $M_{\mathrm{GT}}^{2 \nu}$ have maximum values for equal deformations of both parent

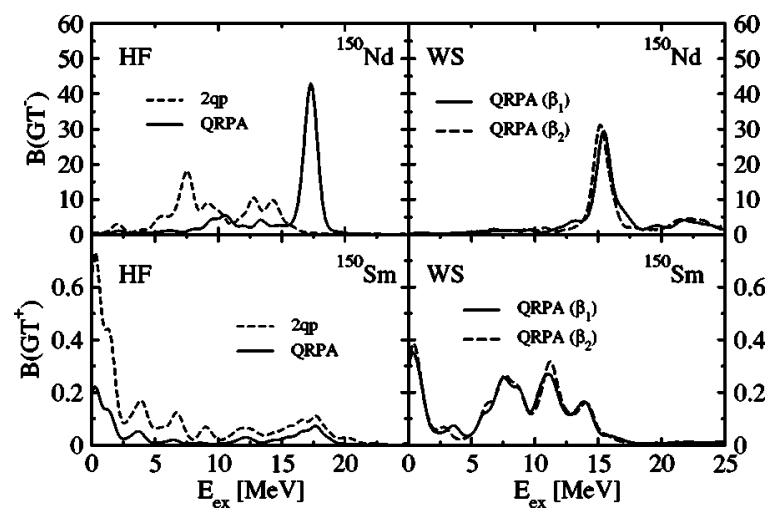

FIG. 14. Same as in Fig. 5 for ${ }^{150} \mathrm{Nd}$ and ${ }^{150} \mathrm{Sm}$.
TABLE IV. Experimental and calculated (HF-Sk3 and WS) summed GT strength. A standard quenching factor 0.6 has been included in the theoretical results.

\begin{tabular}{ccccc}
\hline \hline & & Expt. & HF-Sk3 & WS \\
\hline$\Sigma B\left(\mathrm{GT}^{+}\right)$ & ${ }^{48} \mathrm{Ti}$ & $1.42 \pm 0.2$ & 1.00 & 1.79 \\
& ${ }^{76} \mathrm{Se}$ & $1.45 \pm 0.07$ & 0.48 & 2.06 \\
$\Sigma B\left(\mathrm{GT}^{-}\right)$ & ${ }^{76} \mathrm{Ge}$ & 19.89 & 21.78 & 22.65 \\
& ${ }^{86} \mathrm{Se}$ & 21.91 & 25.34 & 26.09 \\
& ${ }^{100} \mathrm{Mo}$ & 26.69 & 29.14 & 29.93 \\
& ${ }^{116} \mathrm{Cd}$ & 32.70 & 34.79 & 36.41 \\
& ${ }^{128} \mathrm{Te}$ & 40.08 & 43.22 & 43.44 \\
& ${ }^{130} \mathrm{Te}$ & 45.90 & 46.85 & 46.66 \\
\hline \hline
\end{tabular}

and daughter and these values decrease rapidly when the difference between the deformations of parent and daughter increases. In particular, we observe that the $M_{\mathrm{GT}}^{2 v}$ value obtained within a spherical picture $\left(\beta_{\text {parent }}=\beta_{\text {daughter }}=0\right)$ is about the upper limit and only comparable with values obtained with the same deformations for parent and daughter in the deformed picture. As soon as the deformations of parent and daughter change, we get a reduction in the $M_{\mathrm{GT}}^{2 v}$ matrix elements that cannot be obtained from a spherical description. The mechanism of this reduction due to the different deformations was studied in Ref. [12], where it was found that the overlap factor in Eqs. (2.8)-(2.11) is at the origin of the suppression. We can see in Fig. 15 that the experimental values of $M_{\mathrm{GT}}^{2 v}$, plotted as thick segments in each curve, are compatible with particular values of parent and daughter deformations.

We show in Fig. 16 the difference between parent and daughter nuclear quadrupole deformations for the double- $\beta$ emitters. The dots correspond to the results obtained from self-consistent HF calculations with the Skyrme force Sk3, while the extreme values on the vertical segments indicate the maximum and minimum differences compatible with the experimental values in Table II. These are also the extreme values used in WS calculations in Fig. 17.

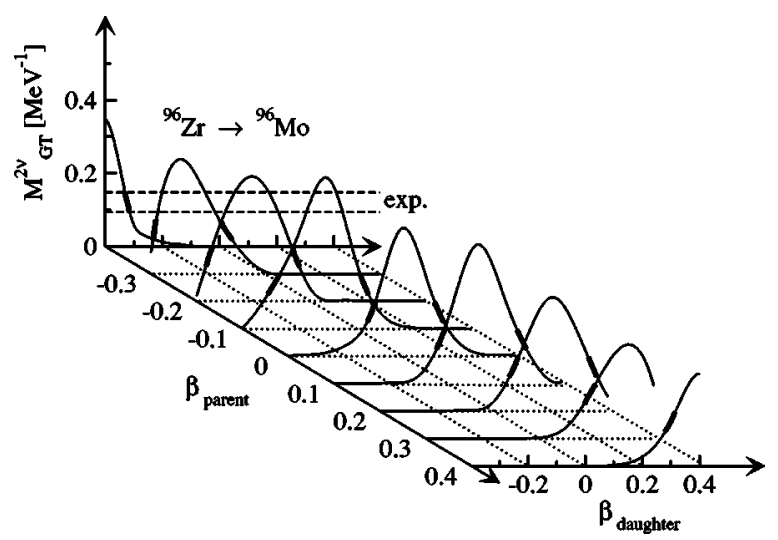

FIG. 15. $2 \nu \beta \beta$-decay matrix elements of ${ }^{96} \mathrm{Zr}$ as a function of both parent and daughter deformations. The two dashed horizontal lines correspond to experimental $M_{\mathrm{GT}}^{2 v}$ extracted from Ref. [34] using $g_{A}=1.0$ and $g_{A}=1.25$. The thick segments in each curve correspond to the experimental values of $M_{\mathrm{GT}}^{2 \nu}$. 


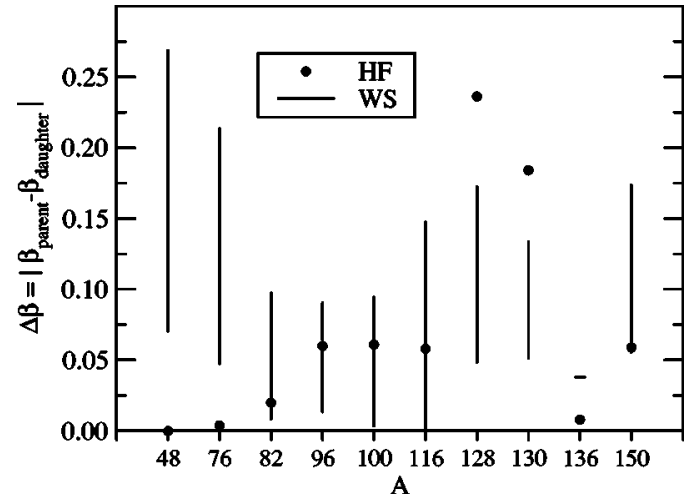

FIG. 16. Difference between parent and daughter quadrupole deformations in double- $\beta$ emitters. Dots are self-consistent results from Skyrme Sk3 calculations. Vertical lines indicate the maximum and minimum experimental differences (see Table II), which are used in WS calculations.

In the next two figures (Figs. 17 and 18) we show the matrix elements $M_{\mathrm{GT}}^{2 \nu}$ for the same double $\beta$ emitters studied in the last section as a function of the particle-particle strength $\kappa_{\mathrm{GT}}^{p p}$. The experimental values extracted by assuming $g_{A}=1.25$ or $g_{A}=1$ are shown by the lower and upper horizontal lines, respectively.

It is well known [4] that the $p p$ interaction introduces a different mechanism of suppression of the $M_{\mathrm{GT}}^{2 \nu}$ matrix elements, which is also interesting to study in our case. In this way, we can compare the effect of the $p p$ force with the effect due to deformation. We first discuss in Fig. 17 the effects of deformation in the WS case by taking the available experimental quadrupole deformations and then we discuss the HF case by considering the self-consistent deformations.

In the WS case (Fig. 17), we use the same potential parameters, gaps, and $p h$ residual interaction as those used in the single $\beta$ calculations in the previous section. For deformations, we take all the experimental possibilities for parent and daughter given in Table II and cross them to calculate $M_{\mathrm{GT}}^{2 \nu}$. Then we show in the figure the upper and lower results obtained as a function of $\kappa_{\mathrm{GT}}^{p p}$ and we draw a shadow region between them. We also show for comparison the results obtained in the spherical case (dashed lines).

The first thing to notice is the already mentioned reduction of $M_{\mathrm{GT}}^{2 \nu}$ as the magnitude of $\kappa_{\mathrm{GT}}^{p p}$ increases, which takes place for both spherical and deformed cases, although the effect of the $p p$ force is larger in the spherical case. The spherical curves decay faster than the deformed ones with $\kappa_{\mathrm{GT}}^{p p}$. This means that the deformed results are more stable (more insensitive) to the particular strength of the $p p$ interaction.

Another interesting feature to mention is that, as expected from the analysis in Fig. 15, deformation introduces in most cases a reduction factor with respect to the spherical result. Only when the deformations of parent and daughter are very similar can the results obtained in the deformed case be larger than the spherical ones. This is, for instance, the case of $A=82$ (see Table II), where the experimental quadrupole deformations [27] are $\beta=0.1944$ in the case of the parent nucleus ${ }^{82} \mathrm{Se}$ and $\beta=0.2022$ in the case of the daughter nucleus ${ }^{82} \mathrm{Kr}$.

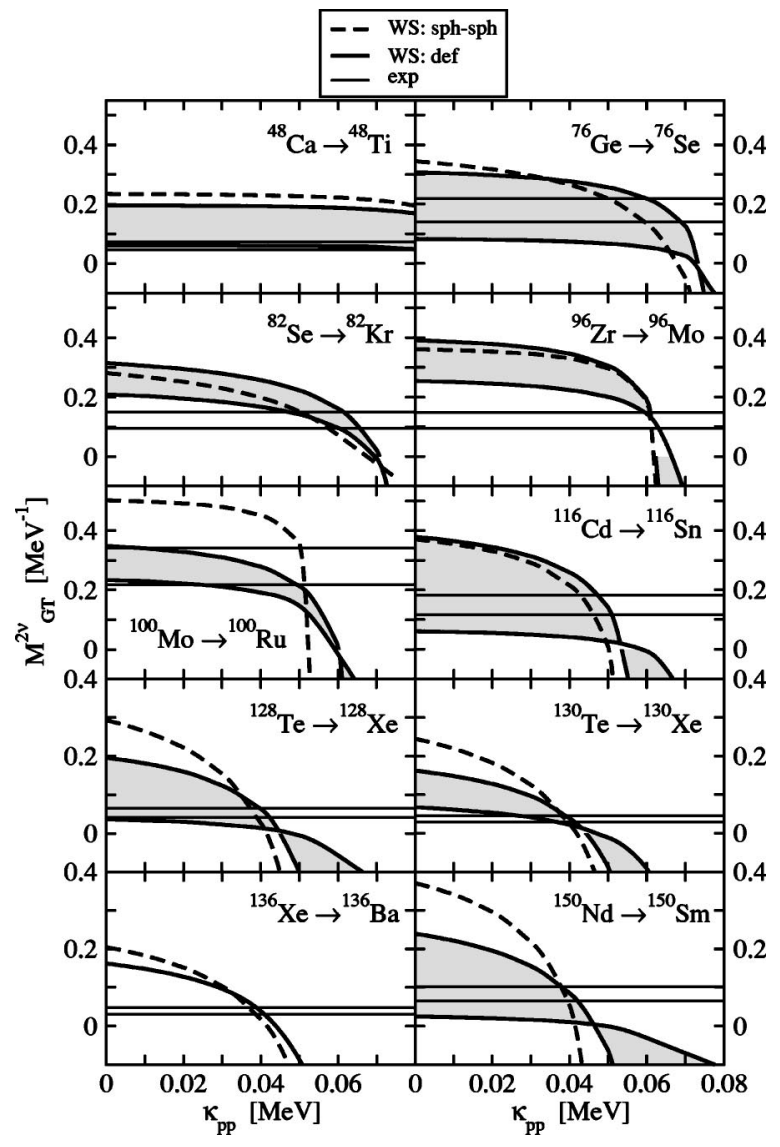

FIG. 17. $2 \nu \beta \beta$-decay matrix elements calculated with WoodsSaxon potentials as a function of the particle-particle interaction strength $\kappa_{\mathrm{GT}}^{p p}$. Dashed lines correspond to the results assuming spherical nuclei. Solid lines correspond to the results obtained by using the maximum and minimum differences between the experimental deformations of parent and daughter (see Table II). Horizontal lines are the experimental $M_{\mathrm{GT}}^{2 \nu}$ extracted from Ref. [34] using $g_{A}=1.0$ and $g_{A}=1.25$.

In Fig. 18, we show the results corresponding to HF calculations with the force Sk3. In this case, the quadrupole deformations are obtained self-consistently (see Table II) and are the same as those used in the previous section to calculate the GT strength distributions in the single $\beta$ decays. The coupling strength of the $p h$ residual interaction has been taken $\chi=0.1 \mathrm{MeV}$ as in the previous section. Contrary to the case of single $\beta$ decay, where the position of the GT resonance is determined by the strength of the $p h$ force and almost independent of the $\kappa_{\mathrm{GT}}^{p p}$ force, we can see in Fig. 18 the sensitivity of the $2 \nu \beta \beta$ decay to the $p p$ force. We find that the experimental values of $M_{\mathrm{GT}}^{2 v}$ are roughly reproduced with values of $\kappa_{\mathrm{GT}}^{p p}=6 / \mathrm{A} \mathrm{MeV}$. This is the reason why we used these values also in the HF calculations of the GT distributions in the previous section.

To illustrate even further the effect of deformation on the $M_{\mathrm{GT}}^{2 \nu}$ matrix elements, we show in Fig. 19 the HF+BCS + QRPA results with the Skyrme force SG2 for the decay ${ }^{96} \mathrm{Zr} \rightarrow{ }^{96} \mathrm{Mo}$ corresponding to the decay of a prolate parent to a prolate daughter or to the decay of a spherical parent into a spherical or a prolate daughter. The actual deformations used in these calculations are those consistently obtained with the 


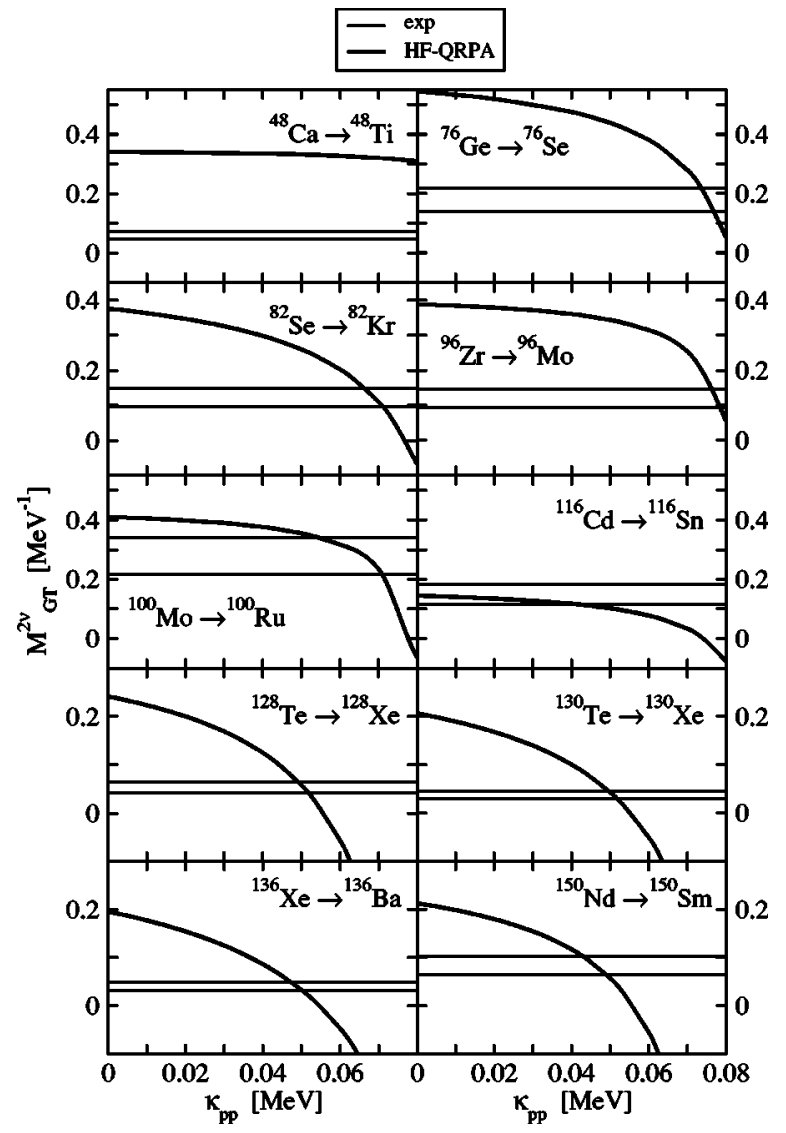

FIG. 18. $2 \nu \beta \beta$-decay matrix elements obtained from Skyrme (Sk3) deformed Hartree-Fock calculations as a function of the particle-particle interaction strength $\kappa_{\mathrm{GT}}^{p p}$. Horizontal lines are the experimental $M_{\mathrm{GT}}^{2 \nu}$ extracted from Ref. [34] using $g_{A}=1.0$ and $g_{A}$ $=1.25$.

force SG2, as can be seen in Table II, namely $\beta=0.016$, 0.147 for the parent ${ }^{96} \mathrm{Zr}$ and $\beta=-0.006,0.119$ for the daughter ${ }^{96} \mathrm{Mo}$. We can see that the transition from the spherical shape to the prolate shape reduces considerably the matrix elements as compared to the spherical/spherical or to the prolate/prolate cases, which are comparable. This reduction, due to the different deformations, makes the results compatible with the experimental values.

\section{CONCLUDING REMARKS}

Using a deformed QRPA formalism, which includes $p h$ and $p p$ separable residual interactions, we have studied the GT strength distributions for the two decay branches $\beta^{-}$and $\beta^{+}$in double $\beta$ decay processes, as well as the two-neutrino double $\beta$ decay matrix elements. In the same manner in which two-neutrino double $\beta$ decay is used to calibrate the nuclear matrix elements for neutrinoless double $\beta$ decay, the single $\beta$ decay branches of parent and daughter are used to test the matrix elements for two-neutrino double $\beta$ decay.

Two different methods, a deformed Skyrme HF approach and a phenomenological deformed WS potential, are used to construct the quasiparticle mean field, which includes pairing correlations in BCS approximation.

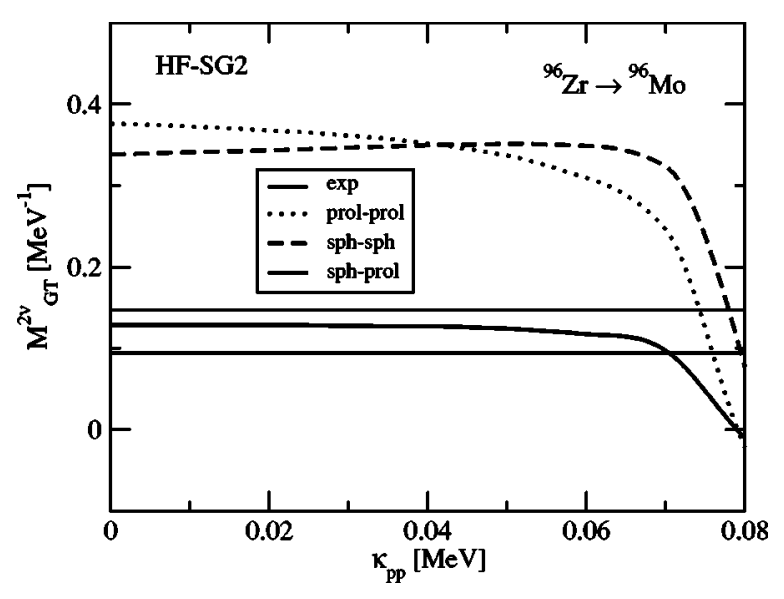

FIG. 19. $2 \nu \beta \beta$-decay matrix elements of ${ }^{96} \mathrm{Zr}$ obtained from Skyrme (SG2) deformed Hartree-Fock calculations as a function of the particle-particle interaction strength $\kappa_{\mathrm{GT}}^{p p}$. The dotted curve corresponds to calculations using the prolate shapes for parent and daughter $\beta_{\mathrm{p}}=0.147, \beta_{\mathrm{d}}=0.119$ (see Table II). The dashed curve is for spherical shapes and the solid curve is for spherical parent and prolate daughter $\beta_{\mathrm{p}}=0.016, \beta_{\mathrm{d}}=0.119$ (see Table II). Horizontal lines are the experimental $M_{\mathrm{GT}}^{2 \nu}$ extracted from Ref. [34] using $g_{A}$ $=1.0$ and $g_{A}=1.25$.

In the case of HF, the deformation is determined selfconsistently and we are able to reproduce the experimental charge radii and quadrupole moments. In the case of WS, the input deformation is taken from experiment and we use two values for each nucleus, one corresponding to the measured quadrupole moment of the first $2^{+}$state and the other extracted from the measured $B(E 2)$ values. The latter can be considered as an upper limit of the $\beta$ value. More experimental work would be needed to improve and complete the experimental determination of the quadrupole moments based on the first of these methods.

Starting from this quasiparticle basis, we perform a pnQRPA calculation with separable forces to obtain the energy distributions of the $\mathrm{GT}^{-}$strength in the parent nucleus and the $\mathrm{GT}^{+}$strength in the daughter nucleus, and from them the $2 \nu \beta \beta$-decay matrix elements. It is well known from previous studies that the $p h$ force allows a reasonable fit of the GT resonance, and that the $p p$ force also affects the $B\left(\mathrm{GT}^{-}\right)$and $B\left(\mathrm{GT}^{+}\right)$distributions. This knowledge has been applied in our paper to test existing parametrizations of the $p h$ and $p p$ residual forces and to see how good agreement can be obtained between experiment and theory with $\mathrm{HF}$ and WS methods. To our knowledge, we have considered for the first time simultaneously all the possible two- $\beta$ emitters and their corresponding daughters comparing their $B(\mathrm{GT})$ to experiment. We find that we need different strengths of the $p h$ force to reproduce the position of the GT resonance, depending on the HF or WS basis. In the first case, a small value of $\chi_{\mathrm{GT}}^{p h}$ $=0.1 \mathrm{MeV}$ reproduces all the measured GT resonances. In the second case, the parametrization obtained in Ref. [8] $\left(\chi_{\mathrm{GT}}^{p h}=5.2 / A^{0.7} \mathrm{MeV}\right)$, using a Nilsson potential, is still valid when using a WS potential. The fact that $\chi_{\mathrm{GT}}^{p h}$ is smaller in $\mathrm{HF}$ than in WS can be understood as arising from the fact that the HF mean field already contains the average effect of spin-spin interaction terms. In both cases, we reproduce rea- 
sonably well not only the position of the resonances but also the total GT strength. It should also be mentioned that the $\mathrm{GT}^{-}$strength of the parent nucleus and the $\mathrm{GT}^{+}$strength of the daughter are located at different energies, a feature that is relevant for double $\beta$ decay because it introduces a reduction of the double $\beta$ decay probabilities. It would be very useful to improve and complete the experimental information on GT strength distributions by $(\mathrm{p}, \mathrm{n})$ and $(\mathrm{n}, \mathrm{p})$ charge exchange reactions on nuclei participating in double $\beta$ decay.

We have also explored the theoretical $Q_{\beta \beta}$ values obtained with the HF method. We find that with the Sk3 force used here, the agreement with the experimental $Q_{\beta \beta}$ is not perfect and that it will be worthwhile to look for a Skyrme force that may optimize agreement with experiment on both GT strengths and $Q_{\beta \beta}$ values. However, taking into account that there is no fitting parameter at all, the agreement between theory and experiment is good. So far, no other approach used to study double $\beta$ decay has been able to obtain $Q_{\beta \beta}$ values so close to experiment. This is an important advantage of the HF method that deserves further exploration.

The effect of deformation on the $2 \nu \beta \beta$-decay matrix elements has been studied first by considering the deformations of both parent and daughter as free parameters. It is found that the matrix elements are suppressed with respect to the spherical case. More precisely, a sizable reduction effect is found that scales with the deformation difference between parent and daughter. This suppression mechanism, which is ignored in spherical treatments, may play an important role in approaching the theoretical estimates to experiment.
In the case of the WS potential, we have studied the $2 \nu \beta \beta$-decay matrix elements by considering the maximum and minimum differences between the experimental deformations of parent and daughter. This procedure generates a region of decaying rates that would be narrowed from an improved experimental determination of the quadrupole deformations.

In the case of HF calculations, we find that using the self-consistent deformations obtained from the minimization of the energy and residual interactions with coupling strengths given by $\chi_{\mathrm{GT}}^{p h}=0.1 \mathrm{MeV}$ and $\kappa_{\mathrm{GT}}^{p p}=6 / \mathrm{A} \mathrm{MeV}$, we are able to reproduce simultaneously the available experimental information on the GT strength distributions of the single $\beta$ branches and the $2 \nu \beta \beta$-decay matrix elements.

\section{ACKNOWLEDGMENTS}

We would like to thank A. A. Raduta for useful discussions. This work was supported by Ministerio de Educación y Ciencia (Spain) under Contract No. BFM2002-03562 and by International Graduiertenkolleg GRK683, by the "Land Baden-Wuerttemberg" within the "Landesforschungsschwerpunkt: Low Energy Neutrinos," by the DFG under 418SPA112/8/03, the VEGA Grant agency of the Slovac Republic under Contract No. 1/0249/03, and by the EU ILIAS project under Contract No. RII3-CT-2004-506222. One of us (R.A.R.) thanks Ministerio de Educación y Ciencia (Spain) for financial support.
[1] J. Bernabeu, Nucl. Phys. B114, 125 (2003); S. M. Bilenky, C. Giunti, J. A. Grifols, and E. Massó, Phys. Rep. 379, 69 (2003).

[2] J. Suhonen and O. Civitarese, Phys. Rep. 300, 123 (1998); A. Faessler and F. Šimkovic, J. Phys. G 24, 2139 (1998); H. V. Klapdor-Kleingrothaus, Sixty Years of Double Beta Decay (World Scientific, Singapore, 2001); S. R. Elliott and P. Vogel, Annu. Rev. Nucl. Part. Sci. 52, 115 (2002); J. D. Vergados, Phys. Rep. 361, 1 (2002); S. R. Eliott and J. Engel, J. Phys. G 30, R183 (2004).

[3] J. A. Halbleib and R. A. Sorensen, Nucl. Phys. A98, 542 (1967).

[4] P. Vogel and M. R. Zirnbauer, Phys. Rev. Lett. 57, 3148 (1986); D. Cha, Phys. Rev. C 27, 2269 (1987); T. Tomoda and A. Faessler, Phys. Lett. B 199, 475 (1987); K. Muto, E. Bender, and H. V. Klapdor, Z. Phys. A 334, 177 (1989).

[5] J. Hirsch, E. Bauer, and F. Krmpotić, Nucl. Phys. A516, 304 (1990); A. A. Raduta, A. Faessler, S. Stoica, and W. A. Kaminski, Phys. Lett. B 254, 7 (1991); J. Toivanen and J. Suhonen, Phys. Rev. Lett. 75, 410 (1995); J. Schwieger, F. Šimkovic, and A. Faessler, Nucl. Phys. A600, 179 (1996); A. A. Raduta, M. C. Raduta, A. Faessler, and W. A. Kaminski, ibid. A634, 497 (1998); A. A. Raduta, F. Šimkovic, and A. Faessler, J. Phys. G 26, 793 (2000).

[6] J. Krumlinde and P. Moeller, Nucl. Phys. A417, 419 (1984).

[7] P. Moeller and J. Randrup, Nucl. Phys. A514, 1 (1990).

[8] H. Homma, E. Bender, M. Hirsch, K. Muto, H. V. Klapdor-
Kleingrothaus, and T. Oda, Phys. Rev. C 54, 2972 (1996).

[9] P. Sarriguren, E. Moya de Guerra, A. Escuderos, and A. C. Carrizo, Nucl. Phys. A635, 55 (1998); P. Sarriguren, E. Moya de Guerra, and A. Escuderos, ibid. A658, 13 (1999); A691, 631 (2001); Phys. Rev. C 64, 064306 (2001).

[10] J. Engel, M. Bender, J. Dobaczewski, W. Nazarewicz, and R. Surnam, Phys. Rev. C 60, 014302 (1999); M. Bender, J. Dobaczewski, J. Engel, and W. Nazarewicz, ibid. 65, 054322 (2002).

[11] P. Sarriguren, E. Moya de Guerra, L. Pacearescu, A. Faessler, F. Šimkovic, and A. A. Raduta, Phys. Rev. C 67, 044313 (2003).

[12] F. Šimkovic, L. Pacearescu, and A. Faessler, Nucl. Phys. A733, 3231 (2004).

[13] P. Sarriguren, E. Moya de Guerra, and R. Álvarez-Rodríguez, Nucl. Phys. A716, 230 (2003).

[14] A. A. Raduta, A. Faessler, and D. S. Delion, Nucl. Phys. A564, 185 (1993); Phys. Lett. B 312, 13 (1993); Nucl. Phys. A617, 176 (1997).

[15] A. A. Raduta, A. Escuderos, A. Faessler, E. Moya de Guerra, and P. Sarriguren, Phys. Rev. C 69, 064321 (2004).

[16] Y. Tanaka, Y. Oda, F. Petrovich, and R. K. Sheline, Phys. Lett. 83B, 279 (1979).

[17] R. Nojarov, A. Faessler, P. Sarriguren, E. Moya de Guerra, and M. Grigorescu, Nucl. Phys. A563, 349 (1993); P. Sarriguren, E. Moya de Guerra, R. Nojarov, and A. Faessler, J. Phys. G 
20, 315 (1994); J. M. Udias, R. Nojarov, and A. Faessler, ibid. 23, 1673 (1997).

[18] M. Beiner, H. Flocard, N. Van Giai, and P. Quentin, Nucl. Phys. A238, 29 (1975).

[19] N. Van Giai and H. Sagawa, Phys. Lett. 106B, 379 (1981).

[20] M. Hirsch, A. Staudt, K. Muto, and H. V. KlapdorKleingrothaus, Nucl. Phys. A535, 62 (1991); K. Muto, E. Bender, and H. V. Klapdor, Z. Phys. A 333, 125 (1989); K. Muto, E. Bender, T. Oda, and H. V. Klapdor-Kleingrothaus, Z. Phys. A 341, 407 (1992).

[21] F. Šimkovic, G. Pantis, and A. Pantis, Phys. At. Nucl. 61, 1218 (1998); Prog. Part. Nucl. Phys. 40, 285 (1998).

[22] H. Flocard, P. Quentin, A. K. Kerman, and D. Vautherin, Nucl. Phys. A203, 433 (1973).

[23] A. Chabanat, P. Bonche, P. Haensel, J. Meyer, and R. Schaeffer, Nucl. Phys. A635, 231 (1998).

[24] H. de Vries, C. W. de Jager, and C. de Vries, At. Data Nucl. Data Tables 36, 495 (1987); G. Fricke, C. Bernhardt, K. Heilig, L. A. Schaller, L. Schellenberg, E. B. Shera, and C. W. de Jager, ibid. 60, 177 (1995).

[25] G. A. Lalazissis, S. Raman, and P. Ring, At. Data Nucl. Data Tables 71, 1 (1999).

[26] P. Raghavan, At. Data Nucl. Data Tables 42, 189 (1989); N. J. Stone (unpublished).
[27] S. Raman, C. H. Malarkey, W. T. Milner, C. W. Nestor, Jr., and P. H. Stelson, At. Data Nucl. Data Tables 36, 1 (1987).

[28] P. Moeller, J. R. Nix, W. D. Myers, and W. J. Swiatecki, At. Data Nucl. Data Tables 59, 185 (1995).

[29] B. D. Anderson, T. Chittrakarn, A. R. Baldwin, C. Lebo, R. Madey, P. C. Tandy, J. W. Watson, B. A. Brown, and C. C. Foster, Phys. Rev. C 31, 1161 (1985).

[30] W. P. Alford, R. L. Helmer, R. Abegg, A. Celler, D. Frekers, P. Green, O. Hausser, R. Henderson, K. Hicks, K. P. Jackson, R. Jeppesen, C. A. Miller, A. Trudel, M. Vetterli, S. Yen, R. Pourang, J. Watson, B. A. Brown, and J. Engel, Nucl. Phys. A514, 49 (1990).

[31] R. L. Helmer, M. A. Punyasena, R. Abegg, W. P. Alford, A. Celler, S. El-Kateb, J. Engel, D. Frekers, R. S. Henderson, K. P. Jackson, S. Long, C. A. Miller, W. C. Olsen, B. M. Spicer, A. Trudel, and M. C. Vetterli, Phys. Rev. C 55, 2802 (1997).

[32] R. Madey, B. S. Flanders, B. D. Anderson, A. R. Baldwin, J. W. Watson, S. M. Austin, C. C. Foster, H. V. Klapdor, and K. Grotz, Phys. Rev. C 40, 540 (1989).

[33] H. Akimune, H. Ejiri, M. Fujiwara, I. Daito, T. Inomata, R. Hazama, A. Tamii, H. Toyokawa, and M. Yosoi, Phys. Lett. B 394, 23 (1997).

[34] A. S. Barbash, Czech. J. Phys. 52, 567 (2002). 